\title{
NÉN ẢNH SỐ THEO NGUYÊN LÝ HÌNH HỌC FRACTAL
}

NGUYẼ̃N TH!̣ HŨ̃U PHƯO'NG

Trường Đại học Mỏ - Địa chất

\section{Tóm tắt:}

Bài báo đề cập đến vấn đề ứng dụng nguyên lý của hình học Fractal vào công nghệ nén ảnh. Từ lâu hình học Fractal đã được ứng dụng trong tạo ảnh máy tính, ứng dụng trong ngành khoa học co bản, đặc biệt là trong công nghệ nén ảnh.. Fractal là phương pháp nén làm mất dữ liệu sử dụng hình học phân hình để đạt đến mức cao hơn của nén ảnh. Công nghệ nén Fractal dựa trên hình ảnh thực của bức ảnh, những phần của bức ảnh giống hệt nhau. Thuật toán Fractal chuyển đổi những vùng này, hay chính xác hơn, hình dạng hình học được chuyển thành dữ liệu toán học gọi là "mã Fractal" để tái tạo lại ảnh đã được mã hóa. Phương pháp nén Fractal có thể thay đổi những giả định đằng sau nén mất và không mất mát dữ liệu. Phương pháp nén ảnh theo nguyên lý hình học Fractal cho tỷ lệ nén cao từ 4:1 đến 10000:1.

\section{Mở đầu}

"Một bức ảnh trị giá bằng ngàn lời giải thích". Hình ảnh là một ngôn ngữ trực quan thể hiện được nhiều ý nghĩa mà lại rất cô đọng. Ngày nay, sự phát triển của lĩnh vực công nghệ thông tin ngày càng mạnh, ảnh số được ứng dụng trong hầu khắp các lĩnh vực của đời sống xã hội và khoa học công nghệ. Xử lý ảnh số là khâu quan trọng trong việc thành lập bản đồ số, bình đồ ảnh số, mô hình số độ cao. Tuy nhiên, ảnh số với trữ lượng thông thường là rất lớn. Vì thế việc nén những thông tin dư thừa trong ảnh là rất cần thiết để có thể lưu trữ thông tin sao cho gọn nhẹ và hiệu quả. Do đặc tính mắt người thường khó nhận biết được hầu hết các chi tiết do đó không cần thiết lưu trữ toàn bộ thông tin ảnh chuẩn mà chỉ cần lưu trữ một tập các phép biến đổi để xấp xỉ thành ảnh ban đầu. Và phép nén Fractal có thể đáp ứng yêu cầu này với một tỉ lệ nén có thể lên đến 10000:1 với độ chính xác khá cao.

2. Cơ sở lý thuyết và phương pháp nghiên cứu

\subsection{Khái niệm hình học Fractal}

Ngày nhận bài: 10/11/2015
Ý tưởng về Fractal nhen nhóm khi Benoit Mandelbrot còn làm việc ở IBM. Nhưng Fractal chỉ thực sự bắt đầu hình thành vào năm 1962, khi ông rời IBM sang Harvard. Năm 1975, cuốn sách "Các đối tượng Fractal" đã được phát hành.

Một Fractal là một vật thể hình học thường có dạng nhấp nhô trên mọi tỷ lệ phóng đại và có thể được tách ra thành từng phần: mỗi phần trông giống như hình tổng thể, nhưng ở tỷ lệ phóng đại nhỏ hơn. Theo định nghĩa của Mandelbrot thì Fractal là "một tập hợp mà trong đó số chiều Hausdorff-Besicovitch lớn hơn số chiều Topo học".

\subsection{Kiến thức cơ sở của hình học Fractal}

Fractal được nghiên cứu như một vật thể toán học. Hình học Fractal là ngành toán học chuyên nghiên cứu các tính chất của Fractal.

Những yếu tố cần quan tâm khi nghiên cứu hình học Fractal như: Số chiều Hausdorff-Besicovitch, Các hệ hàm lặp, Ánh xạ Affine ...

\subsubsection{Số chiều Hausdortt-Besicovitch}

Ngày chấp nhận đăng: 25/11/2015 
Định nghĩa: Cho trước một cấu trúc tự đồng dạng chia thành $\mathrm{N}$ phần, hệ số thu nhỏ của mối phần so với cấu trúc ban đầu là $r$. Ký hiệu $D_{s}$ là đại lượng xác định bởi:

$$
\mathrm{D}_{\mathrm{S}}=\frac{\log N}{\log 1 / r}
$$

Khi đó $D_{S}$ được gọi là số chiều tự đồng dạng của cấu trúc đó.

2.2.2 Các hệ hàm lặp-Iterated Function System (IFS).

Giả sử $(X, d)$ là một không gian metric đầy đủ. Ở đây $X$ được giới hạn bằng $R^{2}$ và $d$ là metric Euclide. Ký hiệu $H(X)$ là không gian các tập con compact khác rỗng của $X$. Ta có các định nghĩa sau:

Định nghĩa 1: Khoảng cách từ một điểm $x \in X$ đến một tập $B E H(X)$ được xác định bởi: $\quad d(x, B)=\min \{d(x, y): y \in B\}$

Định nghĩa 2: Khoảng cách từ một tập $A$ E $H(X)$ đến một tập $B E H(B)$ được xác định bởi: $\quad d(x, B)=\max \{d(x, B): x \in A\}$

Định nghĩa 3: Khoảng cách Hausdorff giữa hai điểm $A$ và $B \in H(H)$ được xác định bởi: $\quad h(A, B)=\max \{d(A, B), d(B, A)\}$

Với các định nghĩa trên ta có định lý:

Định lý về sụ tồn tại của các IFS Fractal:

Ta có $(H(X), h)$ là một không gian metric đầy đủ. Hơn nữa nếu $A_{n} \in H(X)$ với $n=1,2$, ... lập thành một dãy Cauchy thì tập hợp $A$ được xác định bởi:

$$
A=\lim _{0 \rightarrow \infty} A_{n}
$$

cũng thuộc $H(X)$. A có thể được đặc tả như sau;

$A=\left[x \in X: \exists\right.$ một dãy Cauchy $\left[x_{n} \in A\right]$ hội tụ về $X]$

\section{Các hệ hàm lặp Fractal}

Định nghĩa 1: Một hệ hàm lặp gồm một không gian metric đầy đủ $(\mathrm{X}, \mathrm{d})$ và một bộ hữu hạn các ánh xạ co $\mathrm{w}_{\mathrm{n}}$ với hệ số co tương ứng $s_{n}, n=1,2, \ldots, N$. Một IFS được ký hiệu bởi $\left[X: w_{n}, n=1,2, \ldots, N\right]$ và hệ số co $\quad \mathrm{s}=\max _{1 \leq n \leq N} \mathrm{~s}_{\mathrm{n}}$

Định lý sau tóm tắt các kết quả chính của một IFS:

Định lý IFS: Xét một IFS $\left\{\mathrm{X} ; \mathrm{w}_{\mathrm{n}}, \mathrm{n}=1,2\right.$, $\ldots, N$ \} với hệ số co w. Khi đó phép biến đổi W: $H(X) \rightarrow H(X)$ xác định bởi:

$$
\mathrm{W}(\mathrm{B})=\cup_{n=1}^{N} \mathrm{w}_{\mathrm{n}}(\mathrm{B})
$$

Trong đó: $B \in H(X)$ là một ánh xạ co trên không gian metric đầy đủ $(H(X), h(d))$ với hệ số s, tức là:

$$
\mathrm{h}(\mathrm{W}(\mathrm{B}), \mathrm{W}(\mathrm{C})) \leq \mathrm{s} \cdot \mathrm{h}(\mathrm{B}, \mathrm{C}), \forall \mathrm{B}, \mathrm{C} \in H(\mathrm{X})
$$

Ánh xạ này có duy nhất một điểm bất động $A \in H(X)$ với:

$$
\mathrm{A}=\mathrm{W}(\mathrm{A})=\cup_{n=1}^{N} \mathrm{w}_{\mathrm{n}}(\mathrm{A})
$$

và được cho trước bởi $\mathrm{A}=\lim _{n \rightarrow \infty} \mathrm{W}^{\mathrm{n}}(\mathrm{B})$ với bất kỳ $\mathrm{B} \in H(\mathrm{X})$.

Định nghĩa 2: Điểm bất động $A \in H(X)$ mô tả trong định lý IFS được gọi là hấp tử của IFS đó.

\subsection{3. Ánh xạ Affine}

Một ánh xạ affine $\mathrm{f}: \mathrm{R}^{\mathrm{n}} \rightarrow \mathrm{R}^{\mathrm{n}}$ có thể viết dưới dạng $f(X)=A X+b$, trong đó $X$, b là các vector trong $R^{n}$, A là toán tử tuyến tính $R^{n}$. Có thể biểu diễn $A$ bằng một ma trận $\left(a_{i j}\right) i=$ $1,2, \ldots, n ; j=1,2, \ldots, n$.

Tính chất 1: Nếu $\|A\|<1$ thì ánh xạ affine f là co trên $\left(R^{n}, d\right)$ (trong đó $d(x, y)=\|$ $\left.\mathrm{x}-\mathrm{y} \| \forall \mathrm{x}, \mathrm{y} \in \mathrm{R}^{\mathrm{n}}\right)$.

Tính chất 2: Giả sử $\mathrm{A}=\left(\begin{array}{ll}\mathrm{a} & \mathrm{b} \\ \mathrm{c} & \mathrm{d}\end{array}\right)$.

là ma trận của phép biến đổi tuyến tính không suy biến trên $R^{2}$ thì ta có: $A=$ $A_{s} A_{t} A_{u} A_{0}$.

$$
\text { Với } s=(a d-b c) / \operatorname{sprt}\left(c^{2}+d^{2}\right) \text {. }
$$




$$
\begin{aligned}
& t=\left(c^{2}+d^{2}\right) /(a d-b c) \\
& u=(a c+b d) /(a b-b c) . \\
& 0=\arccos \left(d / \operatorname{sqrt}\left(c^{2}+d^{2}\right)\right) .
\end{aligned}
$$

\subsection{Hình học Fractal trong công nghệ nén ảnh}

\subsubsection{Sụ̣ ra đời của phép nén Fractal}

Nén ảnh số là biện pháp giảm kích thước lưu trữ, hiển thị bức ảnh. Ứng dụng của nén ảnh rất rộng rãi từ truyền thông, thông tin đại chúng (TV, video..), văn phòng (Fax..), đến các ngành kỹ thuật, điều tra cơ bản, xử lý ảnh số (đo ảnh, viễn thám, ...). Một trong những mục tiêu quan trọng hàng đầu của công nghệ xử lý hình ảnh hiện nay là sự thể hiện hình ảnh thế giới thực với đầy đủ tính phong phú và sống động trên máy tính. Một ảnh có chất lượng gần như chụp đòi hỏi vùng nhớ 24 bit cho một điểm ảnh, nên để hiện ảnh đó trên màn hình máy tính có độ phân giải tương đối cao như $1024 \times 768$ cấn xấp xỉ 2.25Mb. Với các ảnh "thực" 24 bit, để thể hiện được một hoạt cảnh trong thời gian 10 s đòi hỏi xấp xỉ $700 \mathrm{Mb}$ dữ liệu, tức là bằng sức chứa của một đĩa CD-ROM. Như vậy khó có thể đưa công nghệ multimedia lên $\mathrm{PC}$ vì nó đòi hỏi một cơ sở dữ liệu ảnh và âm thanh khổng lồ. Đứng trước bài toán này, khoa học máy tính đã giải quyết bằng những cải tiến vượt bậc cả về phần cứng lẫn phần mềm. Tất cả các cải tiến đó dựa trên ý tưởng nén thông tin hình ảnh trùng lặp.

Phương pháp nén Fractal được giới thiệu lần đầu tiên bởi M.Barnley vào năm 1987, người đã sáng lập ra công ty chuyên về công nghệ nén ảnh Fractal. Mọi phương thức đều dựa trên phép biến đổi Fractal sử dụng giải thuật hàm lặp. Công nghệ nén Fractal dựa trên hình ảnh thực của bức ảnh, những phần của bức ảnh giống hệt nhau. Thuật toán Fractal chuyển đổi những vùng này, hay chính xác hơn, hình dạng hình học được chuyển thành dữ liệu toán học gọi là "mã Fractal" để tái tạo lại ảnh đã được mã hóa. Phương pháp nén Fractal có thể thay đổi những giả định đằng sau nén mất và không mất mát dữ liệu.

2.2.2. Cách nén hình ảnh theo nguyên lý hình học Fractal

Giả sử chúng ta có ảnh $f$ để mã hóa. Điều này nghĩa là ta mong muốn tìm môt tập các ánh xạ $\mathrm{w}_{1}, \mathrm{w}_{2}, \ldots, \mathrm{w}_{\mathrm{N}}$ với $\mathrm{W}=\bigcup_{i=1} w_{i}$

và $f=|W|$, sao cho $f$ là điểm cố định của toán tử Hutchinson W. Trong trường hợp $w_{i}$ là các ánh xạ co của IFS, đẳng thức điểm cố định: $f=W(f)=w_{1}(f) \cup w_{2}(f) \cup \ldots \cup w_{N}(f)$

gợi ý cách mã hóa theo IFS là có thể thực hiện được. Trong thế giới tự nhiên, mọi vật hiện tượng đều chứa đựng một phần bản chất của Fractal (đặc tính tự tương tự). Tuy nhiên tính tương tự toàn cục thường bị phá vỡ, ta chỉ có thể tìm thấy đặc tính của đó khi phân nhỏ đối tượng ra. Phương pháp mã hóa ảnh Fractal hiểu theo nghĩa đơn giản là đi tìm các cặp $(D, R)$ có đặc tính tương tự mà hợp của các $R$ là toàn miền ảnh. Giả sử ta tìm được cặp $\left(D_{i}, R_{i}\right)$, tức là tồn tại một ánh xạ $w_{\mathrm{i}}$ : $D_{\mathrm{i}} \rightarrow R_{\mathrm{i}}$ sao cho $D_{\mathrm{i}}$ sau khi tác động bởi $w_{\mathrm{i}}$ thì rất gần $\mathrm{R}_{\mathrm{i}}$. Độ gần ở đây được hiểu là khoảng cách Hausdorff giữa chúng là nhỏ.

Như vậy ảnh được phân thành các ô vuông $R_{i}$ và việc đi tìm các $D_{i}$ tương ứng với mỗi $R_{i}$ chính là cốt lõi của vấn đề. Điều này cũng tương đương với việc đi tìm các ánh xạ $w_{i}$ và tập hợp bao gồm các ánh xạ $w_{j}$ tìm thấy được coi là mã Fractal của ảnh đó.

Trên lý thuyết $D_{i}$ và $R_{i}$ có thể phân tách thành các ô vuông từ ảnh gốc theo kích cỡ tùy ý. Tuy nhiên để thuận lợi cho việc trình bày ta chọn cỡ $D_{i}=2 R_{i}$. Các $D_{i}$ được hiểu là các ô nguồn (domain) các $R_{\mathrm{i}}$ được hiểu là các ô đích (range), còn các $w_{\mathrm{j}}$ khi này là các ánh xạ co từ $\mathrm{D}_{\mathrm{i}} \rightarrow \mathrm{R}_{\mathrm{i}}$. 
2.2.3. Thuật toán nén QD (Quadtree Decomposition)

Phương pháp này coi mỗi khối range là một nút trong cây tứ phân. Các khối range trong cùng tầng thì có kích thước như nhau, các khối range ở tầng con có kích thước mỗi cạnh bằng nửa kích thước cạnh của các khối ở tầng cha. Mỗi nút cha có bốn nút con. Số tầng của cây là một số hữu hạn. Thông thường cây tứ phân để nén ảnh Fractal không có nút gốc, mà tầng trên cùng có số nút nhiều hơn một, kích thước khối range của tầng trên cùng sẽ là kích thước để có thể tìm ra $D_{j}$ và $w_{i}$ thỏa mãn trong một số trường hợp và khi đó sẽ tiết kiệm được số ánh xạ và domain phải lưu trữ. Còn các nút lá là các nút đề phòng trường hợp ảnh ít có tính lặp ở các khối range kích thước trên.

Phương pháp được thực hiện thông qua thuật toán như sau:

\section{Minh họa thuật toán}

Procedure Main

$\left\{\right.$ for $I=I_{\min } \ldots I_{\max }$

( for mọi khối range $\mathbb{R}_{\mathrm{j}}^{1} \in \mathbb{R}$.

Phân lớp thành 24 lớp theo các phép đẳng cự)

for mọi khối domain $\mathbb{Z}_{\mathrm{j}}^{\mathbb{1}} \in \mathbb{D}$

Phân lớp thành 24 lớp theo các phép đẳng cự)

for mọi khối range $\mathrm{R}_{\mathrm{i}}^{\operatorname{lmax}} \in \mathrm{R}$

call $\mathrm{QD}\left(\mathrm{R}_{\mathrm{i}}^{\max }\right)$

\}

\section{Procedure $\mathbf{Q D}\left(\mathbf{R}_{\mathrm{i}}{ }^{\prime}\right)$}

$$
\left\{\mathrm{d}_{\min } \leftarrow \infty\right.
$$

for mọi khối domain $D_{j} \in D$ mà $D_{j}$ cùng lớp chính với $\mathrm{R}_{\mathrm{i}}$ for mọi ánh xạ thích hợp với loại của $D_{j}$, $R_{\mathrm{i}}$ 'biến đổi $D_{j}$ xấp xỉ thành $R_{j}^{\prime}$ (trong ánh xạ này) phép đẳng cự đã được chọn trước.

$$
\begin{aligned}
& \left\{\mathrm{d}_{\mathrm{ijk}}=\operatorname{distance}\left(\mathrm{R}_{\mathrm{i}}{ }^{\prime}, \mathrm{w}_{\mathrm{ijk}}\left(\mathrm{D}_{\mathrm{j}}\right)\right)\right. \\
& \text { If } \mathrm{d}_{\mathrm{ijk}}<\mathrm{d}_{\min } \text { then } \\
& \left\{\mathrm{d}_{\min } \leftarrow \mathrm{d}_{\mathrm{j} \mathrm{k}}\right. \\
& \left.\quad \mathrm{w}_{\mathrm{i}} \leftarrow \mathrm{w}_{\mathrm{ij}}\right\} \\
& \} \\
& \text { If } \mathrm{d}_{\text {min }}<\text { ERROR then } \\
& \text { Ghi lại } \mathrm{w}_{\mathrm{i}}
\end{aligned}
$$

Else $\left\{\right.$ If $I>I_{\min }$ then

\{ Chia $\mathrm{R}_{\mathrm{i}}^{\prime}$ thành 4 ô vuông $\mathrm{R}_{\mathrm{i} 0}{ }^{1-1}, \mathrm{R}_{\mathrm{i} 1}{ }^{1-1}$, $R_{\mathrm{i} 2}{ }^{1-1}, \mathrm{R}_{\mathrm{i} 3^{1-1}}$

For count $=0 \ldots 3\}$

\section{Else}

Ghi lại $w_{\mathrm{i}}$ \}

\section{\}}

Sơ đồ khối của thuật toán nén ảnh QD (xem sơ đồ)

\subsubsection{Kết quả nén theo thuật toán $Q D$}

Sau khi thiết kế được thuật toán nén ảnh theo cây tứ phân (QD), ta lập được chương trình nén ảnh theo nguyên lý hình học Fractal. Giao diện chương trình được thể hiện trong hình dưới đây: (xem hình 1, hình 2)

\section{Kết luận}

Phương pháp nén ảnh số theo nguyên lý hình học Fractal có tỷ lệ nén cao, có thể lên đến 10000:1. Phương pháp nén Fractal là phương pháp nén không mất mát dữ liệu, có tỷ lệ nén cao hơn các kỹ thuật nén như JPEG, MPEG, H.261 và H.263.O

\section{Tài liệu tham khảo}

[1]. TS.Trần Đình Trí, PGS.TS.Nguyễn 
Sơ đồ khối của thuật toán nén ảnh $Q D$

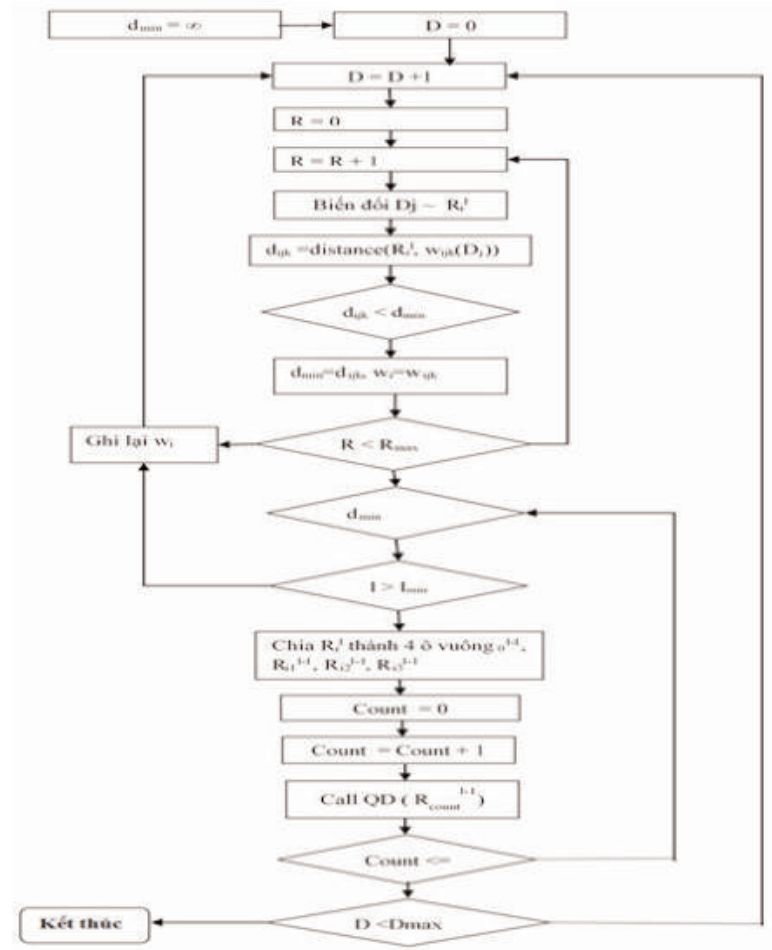

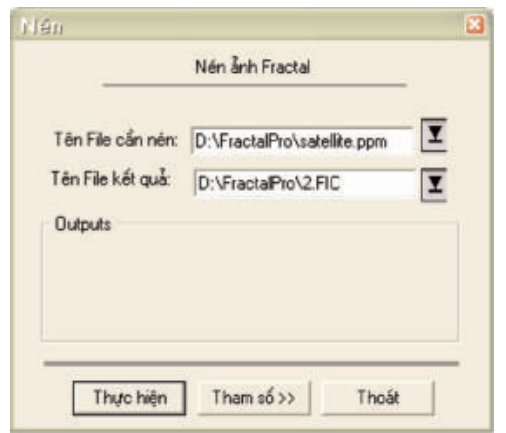

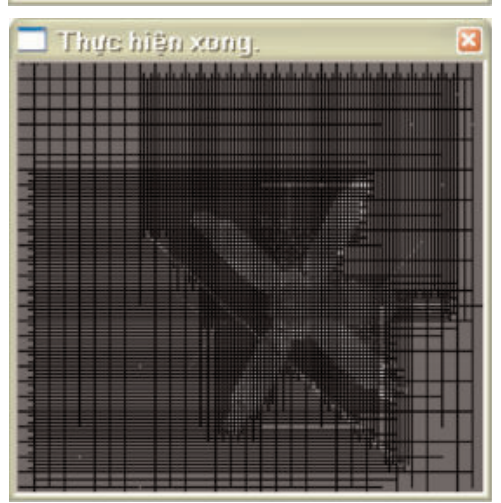

Hình 2: Lựa chọn chức năng nén ảnh và ảnh sau khi nén

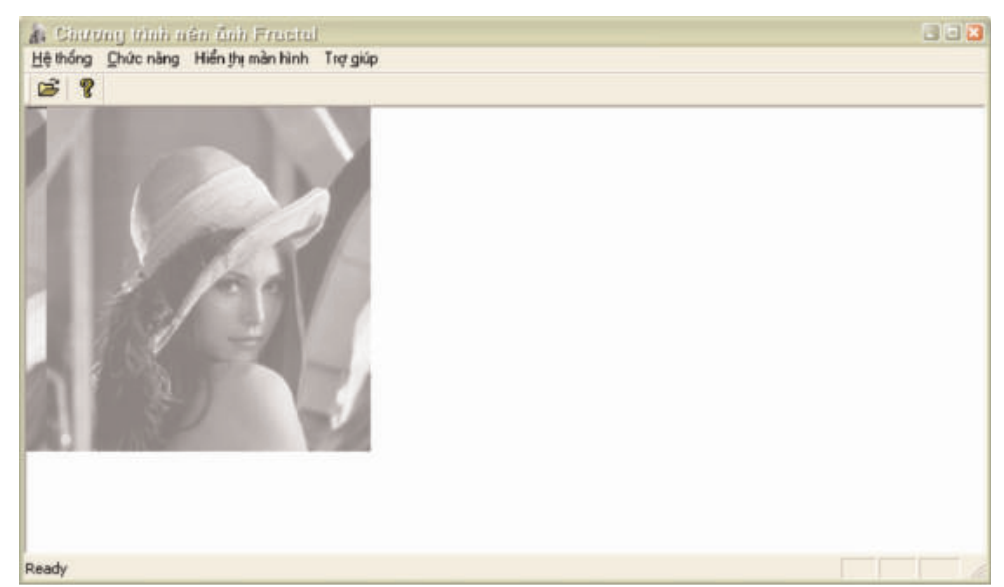

Hình 1: Giao diện chương trình nén ảnh

Trường Xuân. 2006. Đo ảnh giải tích và đo thuật. Hà Nội. ảnh số.

[2]. Lương Mạnh Bá, Nguyễn Thanh Thủy, Nhập môn xử lý ảnh số, Nxb. 2003. Khoa học và kỹ thuật. Hà Nội.

[3]. GS.TSKH. Trương Anh Kiệt, TS. Lê Văn Hường, TS. Trần Đình Trí. 2006 Giáo trình Trắc địa ảnh. Nxb. Khoa học và Kỹ
[4]. Hoàng Tụy. Hình học Fractal (Bài giảng tại viện toán học Hà Nội), tháng 3-4, 2000. Hà Nội.

[5]. Kenneth Folconer, Fractal Geometry Mathematical Foundations and Application. 1993. Great Britaí by Bookcraft Ltd., Midsomer Norton, Avon. 
[6].Website: vi.wikipedia.org/Hinh_hoc

[7].en.wikipedia.org/wiki/Fractal_compression

[8]. Henry Xiao. Fractal Compression.
2004. Queen's University, Kingstion, Ontario, Canada.

[9]. www.fractal-explorer.com/fractalcompression.html. O

\section{Summary}

\section{Digital image compression according to the principle of fractal geometry}

Nguyen Thi Huu Phuong, University of Mining and Geology

The article mentions the application of Fractal geometry in the image compression technology. Fractal geometry has long been used in creating computer images, in basic sciences, especially in the image compression technology. Fractal is a compression method of data loss using the geometric distribution to achieve higher levels of image compression. Fractal compression technology is based on the real images of the picture, whose parts are identical. Fractal algorithms convert these areas, or more accurately, geometric shapes are converted into mathematical data called "fractal code" to reconstruct the image has been encoded. Fractal compression method can change the assumptions behind the compression method of data loss and does not lose any data. Image compression method according to the principle of fractal geometry has high compression ratios from $4: 1$ to $10000: 1$.

\section{MÔ HÌNH CƠ SỞ DŨ๋ LIÊU.....}

(Tiếp theo trang 21)

\section{Tài liệu tham khảo}

[1]. Nguyễn Văn $\mathrm{Ba}$, Phân tích và thiết kế các hệ thống thông tin, Nhà xuất bản Đại học Quốc gia, 2007

[2]. Thạc Bình Cường, Phân tích và thiết kế hệ thống, Nhà xuất bản Thống kê, 2004.

[3]. Đỗ Trung Tuấn, Cơ sở dữ liệu, Nhà xuất bản Đại học Quốc gia, 2004

[4]. Nguyễn Khanh Văn, Giáo trình An toàn \& Bảo mật Thông tin, Đại học Bách Khoa Hà Nội, 2013

[5]. Lê Tiến Vương, Nhập môn cơ sở dữ liệu quan hệ, Nhà xuất bản Khoa học và Kỹ thuật,1996. O

\section{Summary}

\section{A user-authorization database model for webatlas Tay Nguyen}

Nguyen Truong Xuan, Doan Khanh Hoang, Tran Thi Hai Van, Hanoi University of Mining and Geology

Le Thi Kim Thoa, Nguyen Dinh Ky, Institute of Geography - VAST

This paper introduces a design of user-authorization database model for WebAtlas Tay Nguyen application. The design uses the relational data model and database standards level 3. The paper also presents an overview of the MD5 encryption algorithm used in encoding user passwords to enhance system security. $O$ 